\title{
Melanin and lipofuscin as hallmarks of skin aging
}

\author{
Anna Skoczyńska1 ${ }^{1}$ Elżbieta Budzisz ${ }^{1}$, Ewa Trznadel-Grodzka², Helena Rotsztejn ${ }^{3}$ \\ 1Department of Cosmetic Raw Materials Chemistry, Medical University of Lodz, Lodz, Poland \\ ${ }^{2}$ Department of Dermatology and Pediatric Dermatology, Medical University of Lodz, Lodz, Poland \\ ${ }^{3}$ Department of Cosmetology and Aesthetic Dermatology, Medical University of Lodz, Lodz, Poland
}

Adv Dermatol Allergol 2017; XXXIV (2): 97-103

DOI: https://doi.org/10.5114/ada.2017.67070

\begin{abstract}
Discoloration are symptoms of skin aging. They are connected with presence of melanin and lipofuscin, whose excess and abnormal distribution in the skin cause dark spots to appear. Melanin is formed under the influence of tyrosinase during melanogenesis. Its content changes with age, which may be a result of menopause. Lipofuscin is another example of the age pigment. It is composed of proteins, lipids and carbohydrates. It is described as an age pigment because its content increases with age. The formation and accumulation of lipofuscin is inevitable and leads to cell and homeostasis dysfunction because it reduces the proteasome activity.
\end{abstract}

Key words: melanin, lipofuscin, melanogenesis, lipofuscinogenesis, pigments, skin aging.

\section{Melanin}

The color of the skin, eyes and hair depends on melanin. Melanin is composed of polymorphous and multifunctional biopolymers, such as eumelanin (dominant in individuals with dark skin and hair), pheomelanin (dominant in individuals with red hair and skin phototypes I and II, in whom skin tumors are more common), neuromelanin (in human brain) and mixed melanin pigments [1] which are end products of a few-stage transformation of L-tyrosine. Human melanin can be found in the skin, iris, postmitotic cells such as neurons, cardiac myocytes, hepatocytes and glial cells. Melanin is a result of the activity of pigment cells - melanocytes and retinal pigment epithelial cells (RPE) [2], between melanosomes, which contain membrane proteins associated with lysosomes (LAMP) that protect the lysosomal membrane [1]. Melanosomes modify the cellular metabolism because they switch oxidative catabolism to anaerobic glycolysis [1,3], alter NAD/NADH, NADP/ NADPH ratios $[1,4]$ and stimulate the pentose phosphate pathway $[1,5]$. Endosomes are precursors of melanosomes $[6,7]$. Melanin pigments are different in terms of chemical composition, structural and physicochemical properties [1]. Eumelanins are polymorphous, nitrogenous biopolymers; the range of colors starts with black and finishes with brown and they are insoluble in most solvents [1]. They are covalently bonded to proteins. Eumelanins behave as polyanions and are capable to reversibly bind cations, anions, and polyamines [1]. An important feature of eumelanins is a stable paramagnetic state, which is a result of semiquinone units [1]. It also provides a redox activity, mostly towards oxygen species. Pheomelanin in turn is composed of benzothiazine units and its color is yellow to red. It is soluble in alkaline solvents and is a binding factor of proteins, drugs and chemicals $[1,8,9]$. Like eumelanin, it has paramagnetic properties due to additional semiquinone centers $[1,10,11]$. Neuromelanins are macropolymers composed of aminochromes and noradrenalinochromes and their color is brown and black. They are insoluble in organic solvents and demonstrate stable paramagnetic properties. They are able to chelate metals, interact with organic and inorganic compounds [1]. Melanin regulates the biosynthesis of vitamin $D_{3}$ and increases the skin resistance to sunburn and tumors. It is protective as an UV filter and affects thermoregulation, when absorption of solar radiation is changing. The skin contains estrogen, progesterone and androgen receptors. Estrogen receptors are located in keratinocytes, fibroblasts, sebaceous glands, sweat glands, hair follicles, and skin vessels. Progesterone receptors are found in melanocytes and play an important role in the formation of spots, whereas androgen receptors are located in keratinocyte nuclei, hair follicles, and sebaceous glands. These receptors are differently distributed in tissues and the expression of the genes encoding them changes in the course of life.

Address for correspondence: Anna Skoczyńska PhD, Department of Cosmetic Raw Materials Chemistry, Medical University of Lodz, 1 Muszynskiego St, 90-151 Lodz, Poland, phone: +48 4227255 81, e-mail: anna.skoczynska@stud.umed.lodz.pl Received: 30.03.2016, accepted: 27.06.2016. 
Table 1. Comparison of lipofuscin and melanin

\begin{tabular}{|c|c|c|}
\hline \multirow[t]{2}{*}{ Criteria } & \multicolumn{2}{|c|}{ Skin pigments } \\
\hline & Lipofuscin & Melanin \\
\hline Occurrence & $\begin{array}{l}\text { Skin, neurons, cardiac myocytes, retinal epithelial } \\
\text { pigment cells, hepatocytes }\end{array}$ & $\begin{array}{l}\text { Skin, iris, neurons, cardiac myocytes, hepatocytes, } \\
\text { glial cells }\end{array}$ \\
\hline Structure & $\begin{array}{l}\text { Proteins (30-70\%) } \\
\text { Lipids (20-50\%) } \\
\text { Carbohydrates (4-7\%) }\end{array}$ & $\begin{array}{l}\text { Eumelanin - copolymers of DHI and DHICA } \\
\text { Pheomelanin - benzothiazine units } \\
\text { Neuromelanin - aminochromes and } \\
\text { noradrenalinochromes }\end{array}$ \\
\hline Place of formation & Lysosomes & Melanocytes \\
\hline Properties & Autofluorescence & $\begin{array}{l}\text { Redox activity against free radicals due to the } \\
\text { presence of semiquinone }\end{array}$ \\
\hline Importance & $\begin{array}{l}\text { Increased susceptibility to oxidative stress, which } \\
\text { often leads to neurodegenerative diseases, } \\
\text { telomere shortening (acceleration of aging } \\
\text { fibroblasts), inhibition of proteasome activity }\end{array}$ & $\begin{array}{l}\text { Protection from damaging effects of UV radiation, } \\
\text { involvement in the immune response }\end{array}$ \\
\hline $\begin{array}{l}\text { Characteristics of } \\
\text { pigmented skin spots }\end{array}$ & $\begin{array}{l}\text { Brown spots; dark, smooth spots covered with the } \\
\text { epidermis and filled with lipids }\end{array}$ & $\begin{array}{l}\text { Melasma, freckles, post-inflammatory } \\
\text { hyperpigmentation, senile lentigines, UV-induced } \\
\text { pigmentation, pigmentation petaloides actinica }\end{array}$ \\
\hline
\end{tabular}

Hormonal disorders that occur during menopause significantly affect the overall condition and appearance of the skin and its appendages. They manifest themselves with hypo- and hyperpigmentation [3]. There are several types of skin spots being a result of increased melanin synthesis and different deposition (Table 1).

\section{Melanogenesis}

The skin is composed of epidermal units that are responsible for production and distribution of melanin. These units are composed of melanocytes, surrounded by keratinocytes and regulated by the paracrine system. In physiological conditions melanin synthesis occurs in melanocytes. Its enzymatic and structural elements are organized separately in a process resembling lysosome formation [1]. Melanocytes originate in neural crest melanoblasts that migrate to different destinations, such as the epidermis or hair follicles [1]. Tyrosinase presence causes oxidation of tyrosine or L-dihydroxyphenylalanine (L-DOPA) to dopaquinone, initiates eu- or pheomelanogenesis. Tyrosinase can be activated by UV radiation, inflammation, endocrine glands (the pituitary gland, gonads), vitamins $A$ and $P$ P, pantothenic and folic acid. A high concentration of metal ions $\left(\mathrm{Mn}^{2+}\right.$ or $\left.\mathrm{Cu}^{2+}\right)$ also leads to oxidation of L-DOPA to melanins $[1,12,13]$. Inhibition of tyrosinase can be modulated due to the action of vitamin C and endocrine gland activity (adrenal and pineal). Melanocytes are responsible for the production of proopiomelanocortin peptides (POMC), cytokines, nitric oxide (NO), prostaglandins, leukotrienes, which behave in a paracrine or autocrine way in keratinocytes. Furthermore, they are involved in the immune response. Keratinocytes produce several factors (such as POMC, endothelin-1 (ET-1), steel/stem cell factor (SCF), neurotrophins, basic fibroblast growth factor (bFGF), NO) as a result of exposure to ultraviolet radiation, and that can stimulate or inhibit melanogenesis. Migration, proliferation and differentiation of melanocytes depend on mediators that are produced by cells of the dorsal neural tube, i.e. ectoderm, keratinocytes, such as WNT glycoprotein family, endothelin 3 (EDN3), stem cell factor (SCF) [1, $14,15]$. Melanogenesis is a process composed of several steps. Disruption of this process may lead to different types of pigment failures $[1,16,17]$. Understanding of the melanogenesis mechanism allows to explain its causes. In humans between 27 and 30 days of fetal development melanin granules are observed, while melanosomes before week 14 at all stages of maturation [18]. The melanogenesis process is stopped within several weeks, before full pigmentation of cells. Melanosomes mature during several stages $[1,19,20]$. At the beginning, protein matrix is formed in the smooth endoplasmic reticulum and released to cytosol. Then, tyrosinase (enzyme needed in the process of melanin formation) is transferred through vesicular transport from the Golgi apparatus to cytosol. Then, the process of melanin synthesis is activated [18, 20]. In the first step, melanosomes or premelanosomes assume the form of protein matrix, where melanin is deposited (mainly eumelanin). Deposition of melanin and maturation of granules last for another 2 years until melanosomes are fully pigmented [18]. Figure 1 shows a general scheme of melanin biosynthesis. Biosynthesis of melanin is initiated when L-phenylalanine is hydroxylated to L-tyrosine (in in vivo conditions) or directly from L-tyrosine, which is next transformed to L-DOPA. In the next stage L-DOPA is oxidized to dopaquinone. This stage occurs mainly in the course of biosynthesis of eumelanin 


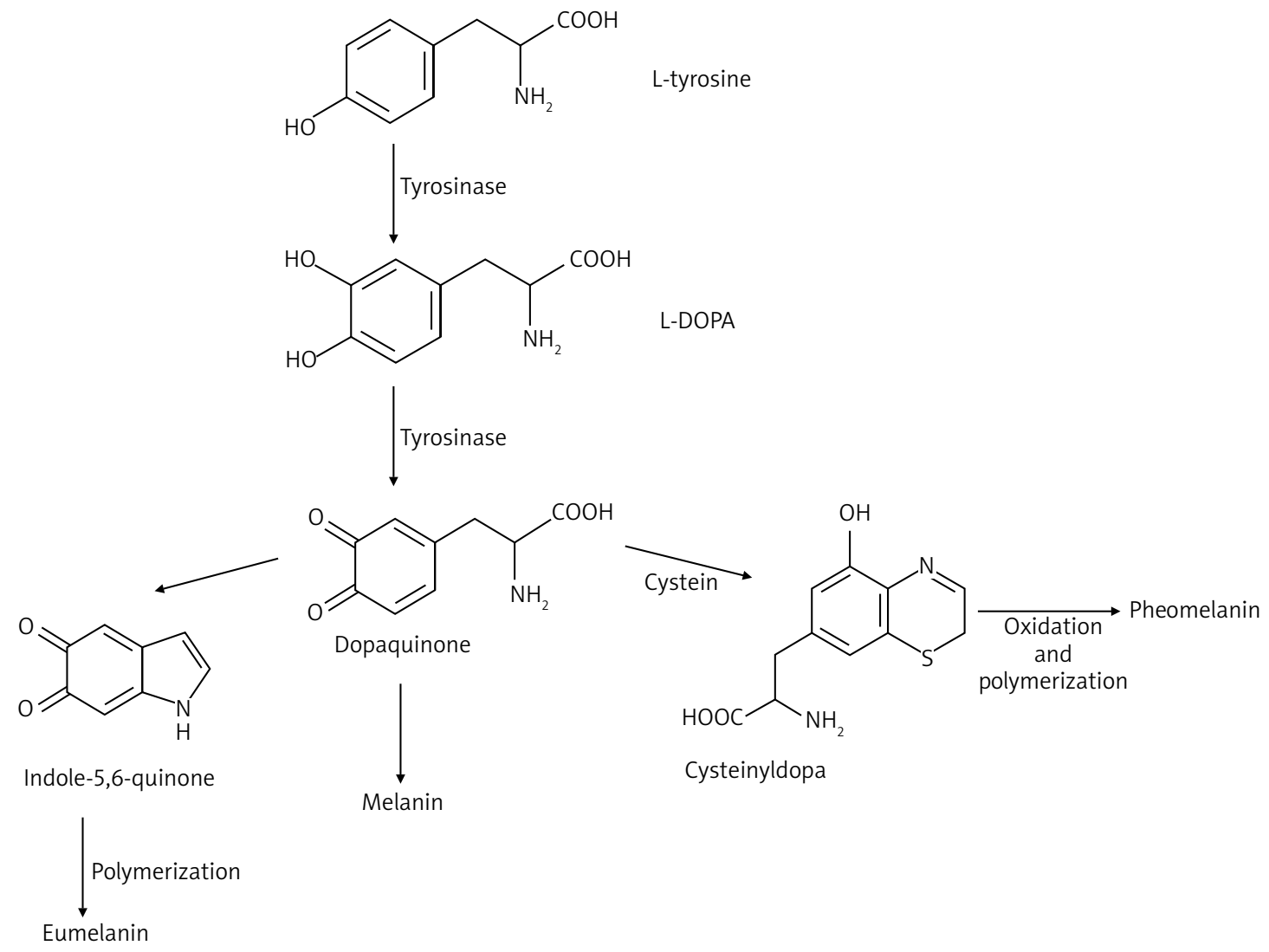

Figure 1. General scheme of melanin biosynthesis

and pheomelanin [1]. Melanosomes formation is a consequence of interactions between rough endoplasmic reticulum (RER) structures, vesicles and channels of the transGolgi Network (TGN). Hence, premelanosomes of the first stage are formed from an outpouching of a smooth membrane [1]. In the second stage of the eumelanogenic pathway, a fibrillar matrix is formed. The eumelanogenic pathway involves transformation of dopaquinone to leukodopachrome, which is preceded by oxidation and reduction reactions. That results in the production of intermediates such us dihydroxyindole (DHI), dihydroxyindole carboxylic acid (DHICA), which are polymerized to eumelanin [1]. There are two ways of maturation of eumelanosomes. Enzymes, which are significant for melanogenesis, are delivered through vesicles to melanosomes that originate from the endoplasmic reticulum and the Golgi apparatus [1]. In the second way tyrosinase and other proteins, involved in melanogenesis, are at first deposited in early developed endosomes through participation of the adaptor protein 3 and then in late developed endosomes. Later on, they connect with melanosomes from the first stage [1, 21]. Melanosomes at the first stage may contain proteins connected with melanogenesis (MRPs), but they are catalytically inactive, until they are released to melanosomes. This process is accompanied by a change of shape of melanosomes and formation of eumelanosomes. Pheomelanogenesis starts with dopaquinone, which is conjugated to cysteine or glutathione. This results in the formation of cysteinyldopa and glutathionyldopa. These products are then converted to pheomelanin [1]. Vitamin E ( $\alpha$-tocopherol) is a potent inhibitor of melanogenesis because it inhibits tyrosinase activity during the post-translational processing. Retinoic acid stimulates maturation of melanocytes. In the basal layer of the epidermis it is able to induce tyrosinase only in white-skinned individuals.

\section{Enzymes of melanogenesis}

Tyrosinase is a glycoprotein that is situated in the melanosome membrane. Its domain is located in the internal membrane, transmembrane and in the cytoplasmic area. It is an enzyme dependent on copper ions and it catalyses transformation of L-tyrosine to L-DOPA. Mutations within a sequence of this enzyme may lead to different diseases connected with disorders of skin pigmentation. A cytoplasmic domain of tyrosinase participates in the transport of the enzyme from the cell nucleus to melanosomes. The internal domain contains a catalytic region (constitutes 90\% of this protein), where 
histidine is located in few residues and binds copper ions $[2,22]$. Tyrosine hydroxylase isoform I is present in melanosomal membrane. It is adjacent to tyrosinase and catalyzes conversion of L-tyrosine to L-DOPA, by initiating tyrosinase activation. Phenylalanine hydroxylase (PAH), dependent on the cofactor 6BH4, catalyzes transformation of phenylalanine to L-tyrosine and also promotes activation of tyrosinase in cytosol [2, 22, 23]. Schallreuter et al. [23] emphasize that these three enzymes are required for melanogenesis [2, 22]. Proteins 1 and 2 are connected to tyrosinase (TRP-1, TRP-2) and are also located in the melanosomal membrane. Their role is not fully explained, but it is possible that TRP-1 plays a role in activation and stabilization of tyrosinase, melanosome synthesis, increases the eumelanin-pheomelanin ratio and also reduces oxidative stress [2, 23, 24]. TRP-2 is also known as dopachrome tautomerase and needs zinc ions for activation. The melanocortin receptor (MC1-R) belongs to the family of $\mathrm{G}$-protein receptors. Its agonists include melanocyte stimulating hormone ( $\alpha-M S H)$, adrenocorticotropic hormone $(\mathrm{ACTH})$, which are cleavage products of proopiomelanocortin (POMC). Agonists of MC1-R activate adenylate cyclase enzyme, which leads to an increase in intracellular CAMP and activation of protein kinase $A$ (PKA). CAMP response element (CREB) is phosphorylated by PKA and it is important for transcription in several genes. Apart from the POMC/MC1-R/CAMP pathway, there are other melanocyte receptors, which are associated with CAMP production and adenyl cyclase. These are muscarinic and $\alpha$ or $\beta$ estrogen receptors. An increase in the estrogen level causes hyperpigmentation during pregnancy. ACTH 1-17/MC1-R and norepinephrine/ $\alpha 1$-adrenergic receptor may cause activation of inositol trisphosphate/diacylglycerol pathway (IP3/DAG), which leads to release of calcium in cytoplasm of melanocytes. diacylglycerol (DAG) activates PKC- $\beta$ and can be released from melanocytes through UVR action in the lipid membrane [2].

\section{Lipofuscin}

Lipofuscin is a waste material of intracellular structures that gets accumulated in lysosomes, between postmitotic cells. It is an undegradable material and cannot be removed dominantly from cells during exocytosis. Postmitotic cells such as neurons, cardiac myocytes, fibers, retinal pigment epithelial cells (RPECS) gradually become brown-yellow and show autofluorescence. Lipofuscin can be detected in neurons of 3- or 4-month old children and in cardiac myocytes of humans in their late childhood. For the first time Hannover $[25,26]$ described such pigment in neurons in 1842, and in 1886 Koneff [25, 27] pointed out its relationship with age. In 1912 Hueck $[25,28]$ introduced the term "lipofuscin", which derived from the Greek word lipo, which means "lipids" and from the Latin word fuscus, which means "dark". Later on, a discovery of lysosomes and lysosomal enzymes made it possible to investigate a relationship between lipofuscin and these organelles [23]. An interference of lipofuscin with cellular recycling processes is supported by the fact that lipofuscin decreases proteasome activity in fibroblasts [23]. It was hypothesized that lipofuscin formation is a result of decreased activity of lysosomal enzymes, mainly cysteine proteases such as cathepsin B, $\mathrm{H}$ and $\mathrm{L}$. An amount of lipofuscin rise with age in postmitotic cells and that is why it is called an age pigment or hallmark of aging [23].

\section{Composition of lipofuscin}

A chemical analysis of lipofuscin granules revealed the presence of protein and lipid compounds (20-50\% and $30-70 \%$, respectively). Protein content consists of different amino acids, while lipid includes triglycerides, free fatty acids, cholesterol, and phospholipids. Carbohydrates account for $4-7 \%$ of lipofuscin content. Metals are also abundant, and iron dominates. Lipofuscin can be detected with the use of histochemical techniques, such as staining for lipids and carbohydrates. Lipids can be stained with Sudan black, Nile blue, $\mathrm{OsO}_{4}$ and carbohydrates with lectins. Lysosomal enzymes associated with lipofuscin granules can be revealed by light and electron immunocytochemistry. Autofluorescence of lipofuscin granules can be observed by fluorescence or electron scanning microscopy with the application of excitation lights of different wavelengths in combination with different barrier filters. Experiments revealed that a reaction between carbonyls and amino compounds produces Schiff bases, 1,4-dihydropyridines, 2-hydroxy-1,2-dihydropyrrol-3-ones, which have autofluorescent properties [25].

\section{Lipofuscinogenesis}

Research data imply there is a relationship between the mechanism of lipofuscin formation and Harman's free-radical theory $[25,29]$, when we bear in mind the role of oxidation reactions in aging. It was hypothesized that free-radical reactions cause damage and polymerization of lipids and proteins, which is an undegradable and fluorescent material. Proteins are the main target of free radicals (ROS) [30, 31]. This type of oxidation increases with age [32-34]. Oxidized proteins are not functional and they are repaired during digestion to peptides or free amino acids in the proteasome or are not removed in the process of exocytosis. With age, these mechanisms weaken. Hence, the oxidized proteins accumulate in cells and cause tissue damage and deposition of lipofuscin $[32,33]$. Cysteine and methionine are most frequently oxidized amino acids. Intrachain aldehydes, ketones and crosslinked connection of cysteine are formed during the oxidation $[35,36]$. Methionine reductases $A$ and $B$ (MSAR/A and MSAR/B, respectively) reduce oxidized me- 
thionine. Other enzymes, such as thioredoxin (TXN) and glutathione reductase (GSR) reduces oxidized cysteine disulfide bridges involving NADPH and cooperate with the MSAR/B [37-40]. Aging and exposure to UV radiation causes dysfunctional recovery mechanisms, thus increasing the level of oxidized protein.

Connections of proteins and other biomolecules is one of results of a ROS attack and probably a reason why phagocytosed material is not degraded $[25,41]$. Brunk et al. $[25,42]$ proposed a scheme of lipofuscin formation in conditions of oxidative stress. Reactive oxygen species, mainly $\mathrm{H}_{2} \mathrm{O}_{2}$, easily defund to lysosomes, which contain various phagocytosed macromolecules, as well as low molecular weight iron. Iron is released from metalloproteins during intracellular degradation. Interactions between $\mathrm{H}_{2} \mathrm{O}_{2}$ and iron lead to $\mathrm{HO}^{\circ}$ formation, which facilitates a formation of cross-linking of surrounding macromolecules and finally lipofuscin. Lipofuscinogenesis gets more intense under the influence of lysosomal proteases which delay degradation of phagocytosed material and need more time for oxidation. Autophagocytosis of mitochondria is the main cause of lipofuscin formation [25] because mitochondria are one of places where ROS are generated so lipofuscin is the aim of their attack [25] and is separated from the cytoplasm by the lysosomal membrane and does not react directly with lysosomal components. An increase in iron between lipofuscin granules may lead to formation of ROS, which causes harmful oxygenation. Moreover, a large number of lysosomes containing lipofuscin may promote cell damage when lysosomal membranes are destabilized by pathogen factors. Fibroblasts containing lipofuscin are more susceptible to apoptosis induced by oxidative stress than cells with lower content of lipofuscin. Examinations of rat neurons revealed that an increase in lipofuscin content was induced by iron. Iron chelators such as desferrioxamine, antioxidants such as vitamin E, glutathione, selenium delay lipofuscin accumulation [25].

The content of melanin may increase or decrease with age. Reduction in melanin concentration in case of an old person can manifest itself mostly with gray hair and this symptom is typical for all people, regardless of gender or race. However, the reduction in melanin is not limited to hair follicles, but also affects the epidermis, the retinal pigment epithelium, certain areas of the central nervous system [43]. Cui et al. [44] demonstrated that activation of p53 occurs during hyperpigmentation, which occurred as a consequence of UV radiation in keratinocytes. The authors concluded that p53 is involved in signaling cytokine receptors paracrine pathway, which promotes the formation of senile lentigines. Their presence is associated with photo-aging and chronological aging. Administration of sex hormones during hormone replacement therapy (HRT) improves the quality and thickness of the skin and also stimulates the function of melanocytes. It leads to an increase in the synthesis of the pigment and causes its uneven distribution. Senile lentigines appear, which may be caused by administration of estrogens and progestogens [3]. Lipofuscin, another marker of aging, is a brownish pigment produced during oxidation of lipoproteins in lysosomes. It has been found that the amount of lipofuscin increases in cells of old bodies [45]. Increased levels of lipofuscin can be assessed by histochemical staining. A higher level of lipofuscin increases susceptibility to oxidative stress, reduces lysosomal degradation, which promotes the development of various pathologies typical for old age. These changes occur in neurodegenerative diseases, such as Alzheimer's and Parkinson's diseases. Large quantities of lipofuscin and lysosomal enzymes are present in amyloid deposits in Alzheimer's patients. It is also believed that it delays the formation of lipofuscin [25]. The content of lipofuscin in postmitotic cells depends on oxidative stress, which causes oxidation of proteins and lipids, which are the structure of lipofuscin. Experiments were performed to determine the correlation between the accumulation of lipofuscin and oxidative stress in fibroblasts of the replication phase. Since studies have confirmed a correlation between telomere shortening and antioxidant efficiency in 22 strains of human fibroblasts, it can be concluded that telomere shortening is an indicator of oxidative stress [46]. Further explanations allow us to conclude that a significant increase in the formation of lipofuscin occurs when the culture almost achieves the replicative senescence. In senescence fibroblasts increase oxidative stress, reduce proteolysis or both of the symptoms appear [46]. The presence of lipofuscin inhibits the activity of the proteasome [46]. The formation of lipofuscin is not a sufficient factor explaining the aging process. It requires interactions with factors that significantly reduce cell growth. The process of shortening of telomeres clearly dominates among the above factors [46]. The presence of lipofuscin in the skin is associated with the appearance of pigmentation generally known as

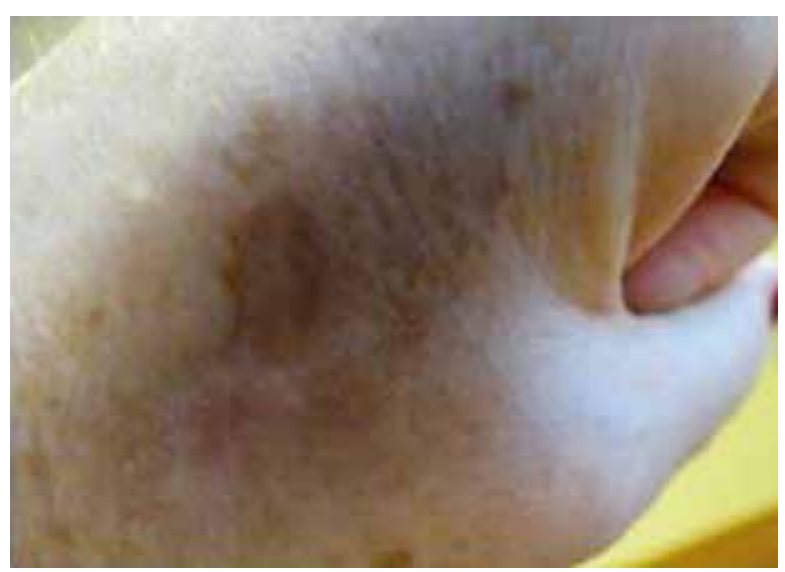

Figure 2. Lipofuscin spots on the dorsal side of hands 
liver spots on the dorsal side of hands (Figure 2), but also face (mainly the cheeks and chest).

These changes may be improved by an application of topical agents for skin lightening. It was also observed that application of high oral doses of vitamin $E$ may reduce the level of lipofuscin in skin biopsies as well as lighten the skin [45].

\section{Acknowledgments}

Financial support from the Medical University of Lodz (grant No. 503/3-066-02/503-31-001 to E. Budzisz and grant No. 503/3-066-01/503-31-001 to H. Rotsztejn, grant No. 502-03/3-066-02/502-34-072 to A. Skoczynska).

\section{Conflict of interest}

The authors declare no conflict of interest.

\section{References}

1. Slominski A, Tobin DJ, Shibahara S, Wortsman J. Melanin pigmentation in mammalian skin and its hormonal regulation. Physiol Rev 2004; 84: 1155-228.

2. Ferreira dos Santos Videira I, Moura DFL, Magina S. Mechanisms regulating melanogenesis. An Bras Dermatol 2013; 88: 76-83.

3. Kubiak M, Rotsztejn H. Wpływ zmian hormonalnych u kobiet na występowanie zaburzeń pigmentacji skóry. Prz Menopauz 2012; 11: 228-32.

4. Scislowski PWD, Slominski A, Bomirski A. Biochemical characterization of three hamster melanoma variants. II. Glycolysis and oxygen consumption. Int J Biochem 1984; 16: 327-31.

5. Scislowski PW, Slominski A. The role of NADP-dependent dehydrogenases in hydroxylation of tyrosine in hamster melanoma. Neoplasma 1983; 30: 239-43.

6. Scislowski PW, Slominski A, Bomirski A, Zydowo M. Metabolic characterization of three hamster melanoma variants. Neoplasma 1985; 32: 593-8.

7. Schiaffino MV. Signaling pathways in melanosome biogenesis and pathology. Int J Biochem Cell Biol 2010; 42: 1094-104.

8. Marks MS, Seabra MC. The melanosome: membrane dynamics in black and white. Nat Rev Mol Cell Biol 2001; 2: 738-48.

9. Boyan BD, Bonewald LF, Sylvia VL, et al. Evidence for distinct membrane receptors for 1 alpha, 25- $(\mathrm{OH})(2) \mathrm{D}(3)$ and24R,25$(\mathrm{OH})(2) \mathrm{D}(3)$ in osteoblasts. Steroids 2002; 67: 235-46.

10. Mars U, Larsson BS. Pheomelanin as a binding site for drugs and chemicals. Pigment Cell Res 1999; 12: 266-74.

11. Sealy R, Hyde J, Felix C, et al. Eumelanins and phaeomelanins: characterization by electron spin resonance spectroscopy. Science 1982; 217: 545-7.

12. Ahene AB, Saxena S, Nacht S. Photoprotection of solubilized and microdispersed melanin particles. In: Melanin, its Role in Human Photoprotection. Zeise L, Chedekel M, Fitzpatrick TB (eds.). Overland Park, KS: Valendmar 1995; 255-69.

13. Prota G. Melanins and Melanogenesis. Academic, New York 1992.

14. Prota G. The chemistry of melanins and melanogenesis. Fortsch Chem Organ Naturst 1995; 64: 93-148.
15. Abdel-Malek ZA, Ross R, Trinkle L, et al. Hormonal effects of vitamin $D_{3}$ on epidermal melanocytes. I Cell Physiol 1988; 136: 273-80.

16. Sealy R, Hyde J, Felix C, et al. Novel free radicals in synthetic and natural pheomelanins: distinction between dopa melanins and cysteinyldopa melanins by ESR spectroscopy. Proc Natl Acad Sci USA 1982; 79: 2885-9.

17. Al Rustom K, Gerard J, Pierard GE. Extrapituitary neuroendocrine melanoderma. Unique association of extensive melanoderma with macromelanosomes and extrapituitary secretion of a high molecular weight neuropeptide related to pro-opiomelanocortin. Dermatologica 1986; 173: 157-62.

18. Double KL, Zecca L, Costi P, et al. Structural characteristics of human substantia nigra neuromelanin and synthetic dopamine melanins. J Neurochem 2000; 75: 2583-9.

19. Boulton ME. Studying melanin and lipofuscin in RPE cell culture models. Exp Eye Res 2014; 126: 61-7.

20. Boulton M. Melanin and the RPE. In: The Retinal Pigment Epithelium. Marmor M, Wolfensberger T (eds). Oxford University Press, New York, Oxford 1998; 68-85.

21. Rozanowska M. Properties and functions of ocular melanins and melanosomes. In: Melanins and Melanogenesis: Biosynthesis, Physiological and Pathological Functions. Brovansky J, Riley PA (eds.). Wiley-Blackwell, Weinheim, Germany 2011; 187-224.

22. Park HY, Kosmadaki M, Yaar M, Gilchrest BA. Cellular mechanisms regulating human melanogenesis. Cell Mol Life Sci 2009; 66: 1493-1506.

23. Schallreuter KU, Kothari S, Chavan B, Spencer JD. Regulation of melanogenesis - controversies and new concepts. Exp Dermatol 2008; 17: 395-404.

24. Kushimoto T, Basrur V, Valencia J, et al. A model formelanosome biogenesis based on the purification and analysis of early melanosomes. Proc Natl Acad Sci USA 2001; 98: 10698703.

25. Brunk UT, Terman A. Lipofuscin: mechanisms of age-related accumulation and influence on cell function. Free Radic Biol Med 2002; 33: 611-9.

26. Hannover A. Mikroskopiske undersögelser af nervesystemet. Kgl Danske Vidensk Kabernes Selskobs Naturv Math Afh (Copenhagen) 1842; 10: 1-112.

27. Koneff H. Beiträge zur Kenntniss der Nervenzellen den peripheren Ganglien. Mitt Naturforsch Gesellsch (Bern) 1886; 44: 13-4.

28. Hueck W. Pigmentstudien. Beitr Pathol Anat 1912; 54: 68232.

29. Harman D. Aging: a theory based on free radical and radiation chemistry. J Gerontol 1956; 211: 298-300.

30. Yaar M, Gilchrest BA. Photoageing: mechanism, prevention and therapy. Br J Dermatol 2007; 157: 874-87.

31. Sander CS, Chang H, Salzmann S, et al. Photoaging is associated with protein oxidation in human skin in vitro. J Invest Dermatol 2002; 118: 618-25.

32. Levine RL, Stadtman ER. Oxidative modification of proteins during ageing. Exp Gerontol 2001; 36: 1495-502.

33. Linton S, Davies MJ, Dean RT. Protein oxidation and aging. Exp Gerontol 2001; 36: 1503-18.

34. Squier TC. Oxidative stress and protein aggregation during biological aging. Exp Gerontol 2001; 36: 1539-50.

35. Grune T, Shringarpure R, Sitte N, Davies K. Age-related changes in protein oxidation and proteolysis in mammalian cells. J Gerontol A Biol Sci Med Sci 2001; 56: B459-67.

36. Rune T, Reinheckel T, Davies KJ. Degradation of oxidized proteins in mammalian cells. FASEB J 1997; 11: 526-34. 
37. Mary J, Vougier S, Picot CR, et al. Enzymatic reactions involved in the repair of oxidized proteins. Exp Gerontol 2004; 39: 1117-23.

38. Holmgren A, Johansson C, Berndt C, et al. Thiol redox control via thioredoxin and glutaredoxin systems. Biochem Soc Trans 2005; 33: 1375-7.

39. Landino LM, Robinson SH, Skreslet TE, Cabral DM. Redox modulation of tau and microtubule-associated protein- 2 by the glutathione/glutaredoxin reductase system. Biochem Biophys Res Commun 2004; 323: 112-7.

40. Zhang H, YM Go, Jones DP. Mitochondrial thioredoxin-2/peroxiredoxin-3 system functions in parallel with mitochondrial GSH system in protection against oxidative stress. Arch Biochem Biophys 2007; 465: 119-26.

41. Kikugawa K, Kato T, Beppu M, Hayasaka A. Fluorescent and cross-linked proteins formed by free radical and aldehyde species generated during lipid oxidation. Adv Exp Med Biol 1989; 266: 345-57.

42. Brunk UT, Jones CB, Sohal RS. A novel hypothesis of lipofuscinogenesis and cellular aging based on interactions between oxidative stress and autophagocytosis. Mutat Res 1992; 275: 395-403.

43. Gilchrest BA, Eller MS, Yaar M. Telomere-mediated effects on melanogenesis and skin aging. I Investig Dermatol Symp Proc 2009; 14: 25-31.

44. Cui R, Widlund HR, Feige E, et al. Central role of p53 in the suntan response and pathologic hyperpigmentation. Cell 2007; 128: 853-64.

45. Georgakopoulou EA, Tsimaratou K, Evangelou K, et al. Specific lipofuscin staining as a novel biomarker to detect replicative and stress-induced senescence. A method applicable in cryo-preserved and archival tissues. Aging (Albany NY) 2013; 5: 37-50.

46. Sitte N, Merker K, Grune T, von Zglinicki T. Lipofuscin accumulation in proliferating fibroblasts in vitro: an indicator of oxidative stress. Exp Gerontol 2001; 36: 475-86. 